\title{
Active biological mechanisms: transforming energy into motion in molecular motors
}

\author{
William Bechtel $^{1}$ D . Andrew Bollhagen ${ }^{1}$
}

Received: 29 October 2019 / Accepted: 3 August 2021 / Published online: 10 August 2021

(C) The Author(s) 2021

\begin{abstract}
Unless one embraces activities as foundational, understanding activities in mechanisms requires an account of the means by which entities in biological mechanisms engage in their activities - an account that does not merely explain activities in terms of more basic entities and activities. Recent biological research on molecular motors (myosin and kinesin) exemplifies such an account, one that explains activities in terms of free energy and constraints. After describing the characteristic "stepping" activities of these molecules and mapping the stages of those steps onto the stages of the motors' hydrolytic cycles, researchers pieced together from images of the molecules in different hydrolyzation states accounts of how the chemical energy in ATP is transformed in the constrained environments of the motors into the characteristic activities of the motors. We argue that New Mechanism's standard set of analytic categories - entities (parts), activities (operations), and organization-should be expanded to include constraints and energetics. Not only is such an expansion required descriptively to capture research on molecular motors but, more importantly from a philosophical point of view, it enables a non-regressive account of activities in mechanisms. In other words, this expansion enables a philosophical account of mechanistic explanation that avoids a regress of entities and activities "all the way down." Rather, mechanistic explanation bottoms out in constraints and energetics.
\end{abstract}

Keywords Mechanistic explanation · Activities · Energetics · Constraints · Molecular motors

This article belongs to the topical collection "Multiscale Modeling and Active Materials", edited by Patrick McGivern and Nick Brancazio.

William Bechtel

bechtel@ucsd.edu

Andrew Bollhagen

abollhag@ucsd.edu

1 Department of Philosophy, University of California, San Diego, La Jolla, CA 92093-0119, USA 
In recent years, interest in biological motion has blossomed because of the realization that much of cell behavior and architecture depends on the directed transport of macromolecules, membranes, or chromosomes within the cytoplasm. Indeed, modern microscopy has transformed our view of the cell interior from a relatively static environment to one that is churning with moving components, not unlike the bustling traffic in a metropolitan city. (Vale \& Milligan, 2000).

\section{Introduction}

According to the New Mechanist philosophy of science, explaining a phenomenon involves specifying the organized entities composing the mechanism responsible for it. Importantly, the entities in a mechanism are active and a specification of the activities in which they engage is necessary for understanding how the mechanism undergoes characteristic changes that produce the phenomenon it explains. As Machamer et al. (2000) (henceforth, MDC) write:

[I]t is . . impoverished to describe mechanisms solely in terms of entities, properties, interactions, inputs-outputs, and state change over time. Mechanisms do things. They are active and so ought to be described in terms of the activities of their entities, not merely in terms of changes in their properties. (p. 5)

Drawing from a specification of the mechanism responsible for the action potential in neurons, MDC's list of activities includes, to name only a few, "fitting, turning, opening, colliding, bending and pushing..."(Machamer et al., 2000). Other mechanists agree. Consulting biochemistry textbooks, Illari and Williamson (2013) list "trigger, binding, phosphorylates, modifying, wrapping... unwinding, supercoiling... and stabilizing" as terms denoting activities.

There is some debate, however, over whether activities, as an ontological category, are required or even helpful in understanding mechanisms. Some, like Cartwright (1999), argue against including activities in our ontology and favor capacities, "The knowledge we have of the capacity of a feature is not knowledge of what things with that feature do [activities] but rather knowledge of the nature of the feature." Others, like Machamer (2004), insist on activities, "One can't specify a... capacity without having some way to identify what the capacity does when it is actualized or exercised. However, being able to recognize what a capacity does when actualized or the activity that constitutes it presupposes having the concept of activity."

Our focus here, however, is not on the metaphysical issue of whether and how to incorporate activities into the ontology of science but with the question of how to explain activities. For those concerned with ontology, the fact that scientists assert that the alpha helix in a sodium channel rotates in response to the spreading depolarization of the axon argues that "rotatings" need ultimately to show up in a "descriptive ontology of science." 1 This leaves the question: by what means does

\footnotetext{
1 We borrow this phrase from Illari and Williamson (2013).
} 
this rotation occur? More generally, by what means do the entities in a mechanism engage in their activities? From this point of view, merely attributing to activities a positive ontological status is unilluminating. As Winning and Bechtel (2018) write, "The activity of a mechanistic component is what the component actually does; it is not the why. For this reason, we contend that what is needed is an account... of whatever it is about mechanisms... in virtue of which activity is brought about." We follow them here in thinking that simply countenancing activities in our ontology does not help us understand activities in mechanisms. To do so is to merely re-state, in a round-about (and fruitlessly reifying) way, the fact that mechanisms are active. What is important is to give an account of the means by which they are.

We cannot make progress in addressing this question by engaging in a priori speculation. Just as the New Mechanists in philosophy of science developed their accounts of mechanisms by examining actual science (Bechtel \& Richardson, 1993/2010; Bechtel \& Abrahamsen, 2005; Machamer et al., 2000; Craver \& Darden, 2013; Glennan \& Illari, 2018), we need to proceed by examining instances in which scientists have had success in addressing this question. Like the New Mechanists, we focus on examples from biology. Fortunately, there are a number of examples in recent biology in which researchers have addressed just this question-they have not only garnered evidence for the occurrence of certain types of activities but have also offered explanations of these activities. To do so they have had to go beyond decomposing the mechanism into constituent entities performing their activities, since there is little gain, when the question is to explain how activities are active, in explaining one activity in terms of others. In order to avoid a regress of activities-to avoid the conclusion that mechanistic explanations always explain an activity in terms of others-one needs an account of the means by which mechanisms are active that does not itself appeal to the category of activities.

A key component of these accounts of biological activities is identifying the source of Gibbs free energy that is utilized in the activity. Although a focus on the source of free energy has notably been lacking from the various characterizations of mechanisms and mechanistic explanation, a basic principle from physics that is honored in biology is that no work can be performed without a source of free energy. The challenge in explaining activities is twofold - to identify the source of freeenergy and to understand how that free-energy is converted into a specific activity. At a generic level, as argued by Winning and Bechtel (2018), the latter depends on how the constituent parts or entities of a mechanism constrain the flow of free energy into the performance of a particular activity. This is familiar in the case of human-made machines such as a car: the free energy released in the combustion of gasoline is constrained to generate mechanical motion by exerting force on a piston which is then passed, through the driveshaft, to the wheels. In this instance, heat is the intermediary, but in living organisms heat is recognized as a waste-product-its diffusion is not constrained to produce work. How, then, in biological mechanisms, is free energy constrained to produce work? Understanding that will provide a foundation for understanding how mechanisms in biological organisms are active. ${ }^{2}$

\footnotetext{
2 The relation of entities or activities to sources of free energy parallels Klein's (2018) characterization of the relation between mechanisms and resources. On Klein's account, resources may either be available or not, are not individually important, potentially interact promiscuously with the parts of a mechanism,
} 
To address the question of how free energy is transformed into activity in biological mechanisms, we focus on a particular class of cellular mechanisms, molecular motors that convert free energy, in the form of ATP, into the exertion of force, either on objects external to the cell or other components of the cell. For this paper we limit ourselves to two molecular motors, myosin II (hereafter, myosin), which pulls itself along actin fibrils, resulting in contraction of muscles, and kinesin-1 (hereafter, kinesin), which pulls cargo toward the periphery of a cell along microtubules. ${ }^{3}$ In many respects, research on these motors follows the familiar picture of mechanistic research: scientists identified phenomena of interest and then decomposed the responsible mechanism to show how it generates the phenomenon. But there are important differences between these endeavors and those that have been the focus of the New Mechanists. First, a key element was describing how free energy, in the form of ATP, figures in the operation of the motor. Motors are ATPases-enzymes that break the bond between the third $(\gamma)$ phosphate group and the rest of the molecule, releasing free energy. The challenge was to link the stages of the molecules' hydrolytic activity with the stages of their mechanical movements. We develop this in Sect. 2. However, as we discuss in Sect. 3, researchers' explanatory efforts did not stop there. Rather, taking these activities as phenomena to be explained, they developed accounts of how the free energy released by ATP hydrolysis in the constrained environment of the molecules results in their characteristic activities-stepping along actin filaments, in the case of myosin, and along microtubules for kinesin. Importantly, these accounts are not given in terms of further entities and activities in a mechanism at a lower explanatory level. Rather, they are given in terms of constraints and energetics. Section 4 fleshes out the account and suggests that the lesson we extract for the case of molecular motors research generalizes to mechanisms other than molecular motors. Biological mechanisms and their components are indeed active, but this activity is explained in terms of the constrained release of free energy. We conclude that while the motors we discuss constitute "bottom-out entities" (the term was introduced by MDC) for mechanistic explanation in biology, analysis of mechanisms at higher levels can benefit from construal in terms of

\footnotetext{
Footnote 2 (continued)

but are nonetheless essential for the functioning of a mechanism. Klein applies his account of a resource to gasoline, the form of free energy for a car, but these features are true of sources of free energy in general. Winning and Bechtel's analysis shows why free energy is a needed resource and how it figures in the understanding of the mechanism - the activities performed by the mechanism result from free energy being constrained by the components of a mechanism. This points, though, to an important difference: for Klein, resources are the patient of action, whereas on Winning and Bechtel's account, free energy is the source of activity in a mechanism.

3 Myosins and kinesins constitute a natural category since both are P-Loop ATPases that are very similar to G-proteins (Kull et al., 1998). There are several other classes of molecular motors that hydrolyze ATP to generate motion, such as dyneins. Neither myosin nor kinesin designates a single protein. There are, in fact, at least 35 different classes of myosins, 13 of which have members occurring in humans. Although it was the first discovered myosin, muscle myosin is now designated myosin II. After the discovery of the first kinesin, 14 different classes of kinesins have been identified. The originally discovered kinesin, sometimes referred to as conventional kinesin, is a member of the kinesin-1 family. We will focus on myosin II and conventional kinesin, appealing to research on other myosins and kinesins as it contributed to the understanding of myosin II and conventional kinesin.
} 
energetics and constraints. Identifying how free energy is constrained, however, is not, strictly speaking, necessary as higher-level mechanistic explanations can simply appeal to entities and activities. Recognizing that one can further explicate activities in terms of constrained release of free energy dispels the metaphysical mystery that results when activities are construed as ontologically fundamental. Section 5 summarizes what we claim to have accomplished.

\section{Characterizing the role of free energy in molecular motor movement}

In this section, we describe how researchers developed accounts of the mechanisms responsible for muscle contraction and axonal transport. In both cases, the relevant mechanisms are molecular motors-myosin and kinesin respectively. The research involved identifying the motors, characterizing the movements-activities-of their parts, and mapping the stages of these movements onto stages of the motors' hydrolytic cycles. At this stage, researchers had identified the mechanisms for the phenomena of interest and specified them in terms of their entities and activities. But, as we will see in Sect. 3, their explanatory efforts did not stop there. Rather, researchers developed accounts of the means by which these mechanisms engaged in their activities according to which the release of free energy due to hydrolysis in the molecules' constrained environment results in their characteristic stepping activities. Importantly, this further account was not given in terms of still more entities and activities but rather in terms of constraints and energetics.

\subsection{Identifying ATP hydrolysis in the operation of the myosin motor}

Leeuwenhoek initiated the microscopic examination of muscle, but it was with the much-improved microscopes and advent of staining techniques in the mid-nineteenth century that researchers advanced the now classic descriptions of anisotropic (A) and isotropic (I) bands on muscle fibers and described how I bands shortened while A bands moved closer together when muscles contracted. During the same period Kühne (1864) isolated from muscle-press juice when the muscle was in the rigor state what he took to be a single viscous molecule he called myosin. Subsequent researchers attributed myosin to normally functioning muscles and proposed that muscle contraction resulted from changes in myosin. In the wake of the discovery of adenosine triphosphate (ATP) and characterization of it as an energy source for biological activities, Engelhardt and Ljubimowa (1939) established that myosin functioned as an ATPase. In research conducted during World War II but only published in a widely accessible venue afterwards, Szent-Györgyi (1945) showed that what had been taken to be one molecule actually consisted of two proteins, myosin and actin. Both actin and myosin constitute filaments, but only myosin functions as an ATPase (for detailed historical accounts, see Needham, 1971; Huxley, 1977; Rall, 2014).

Before researchers could establish how ATP hydrolysis figured in muscle contraction, they needed to discover how myosin moved with respect to actin. A pair 


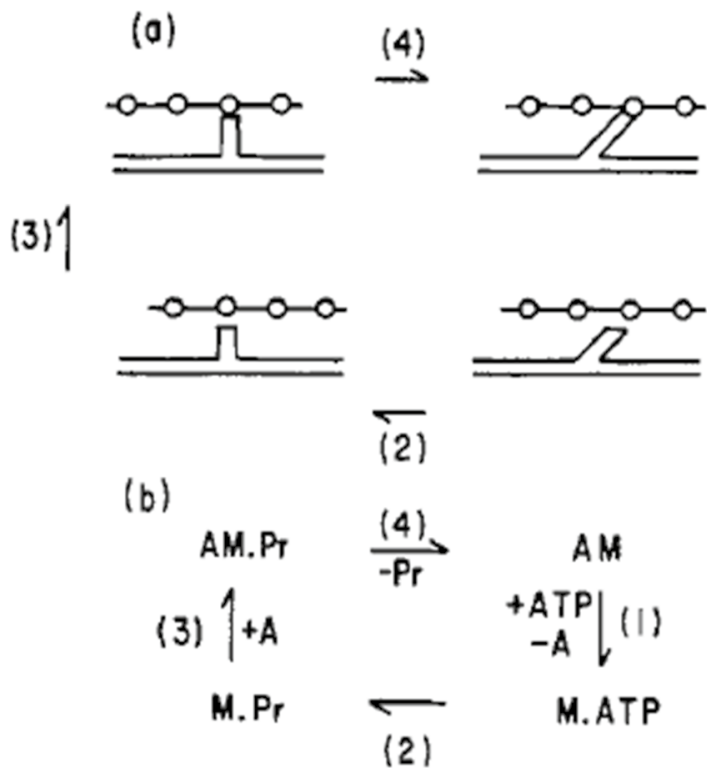

Fig. 1 a The stages in the crossbridge cycle in which (1) myosin disconnects from actin, (2) changes orientation, (3) rebinds actin at a new position, and (4) exerts force moving the actin in the powerstroke. b Corresponding stages of the Lymn-Taylor cycle. Reprinted with permission from Biochemistry, 10, Lymn, R. W., \& Taylor, E. W., Mechanism of adenosine triphosphate hydrolysis by actomyosin, Fig. 7. Copyright 1971, American Chemical Society

of papers published back-to-back in 1954, one by Huxley and Niedergerke (1954) using interference microscopy and one by Huxley and Hanson (1954) using X-ray crystallography and electron microscopy, revealed a critical feature: crossbridges between thin (actin) and thick (myosin) filaments. To characterize the activity of these crossbridges, researchers had to procure multiple images that showed the cross-bridges in different states of muscle contraction. From such evidence, Huxley $(1958,1969)$ articulated the crossbridge hypothesis (shown in Fig. 1a) according to which a bridge that projects from myosin to actin goes through a cycle of stages in which it detaches from actin (1), moves (2), reattaches to actin (3), and exerts force in a "powerstroke" to pull itself along the actin filament (4). ${ }^{4}$

Independently, biochemists Lymn and Taylor (1971) added a radioactive tracer into the third phosphate group of ATP and followed the process of ATP hydrolysis by myosin. This revealed both rapid hydrolysis of ATP to ADP and Pi and a much slower release of Pi unless myosin is bound to actin. They mapped these steps onto the steps in the movement of the crossbridge in what came to be known as the Lymn-Taylor cycle (Fig. 1b). In the first step myosin binds ATP and detaches from actin (1). As ATP is hydrolyzed the crossbridge returns to a right angle (2); it then binds to a new locus on myosin (3). Myosin remains bound to the reaction products

\footnotetext{
${ }^{4}$ See Hitchcock-DeGregori and Irving (2014) for a detailed analysis of Hugh Huxley’s contributions.
} 
(Pr), ADP and Pi, until step 4, at which their release corresponds to a movement of the crossbridge.

Integrating the crossbridge hypothesis and the Lymn-Taylor cycle yielded a coherent description of the relation of ATP hydrolysis to myosin movement that, in its basics, has been adopted in subsequent research and significantly elaborated on. The key force-applying step that causes the myosin filaments to pull themselves along actin filaments is known as the powerstroke (step 4 in Fig. 1). A key feature of the account is that while hydrolysis of ATP provides the energy for the powerstroke, ATP is not directly involved in that step. The powerstroke occurs with the release of ADP and Pi that had resulted from hydrolysis in step 2. At the time of hydrolysis, the crossbridge is not attached to actin. Only after myosin rebinds to actin and successively expels Pi and ADP does the powerstroke occur which causes the crossbridge to change conformation and move approximately $10 \mathrm{~nm}$ with respect to actin. Myosin remains tightly bound to actin (this is known as the rigor state as it was assumed to correspond to the state assumed in rigor mortis) until it binds to another ATP molecule, whereupon it detaches from actin.

\subsection{Identifying ATP hydrolysis in the operation of the kinesin motor}

Kinesin was only discovered in 1985 based on two lines of research. The first identified microtubules as fibrils within axons in electron micrograph studies (De Robertis \& Franchi, 1953; Palay, 1956). In subsequent research these were shown to consist of tubulin molecules that self-organize in a polar fashion, with what is identified as the +- end pointing away from the cell center. The second, relying on radioactive tracers, revealed the transport of proteins and larger structures along axons (see Grafstein \& Forman, 1980, for a review). Combining techniques of video and differential interference contrast microscopy, Allen and his collaborators (Allen et al., 1981, 1982) showed that this transport occurred along microtubules in both directions, with the cargo sometimes falling off and remaining motionless until another microtubule "came along to provide a substrate or carrier for the movement." Adams (1982) and Lasek and Brady (1984, 1985) showed that movement along microtubules depended on ATP. To identify the responsible ATPase protein, Vale et al. (1985) centrifuged squid axoplasm, suspended the resulting particle in ATPcontaining buffer, and added the product to microtubules placed on a glass coverslip. Observing movement of carboxylated latex beads along the coverslip and noting that the molecular weight of this particle was distinct from both myosin and dynein (the two molecular motors then known), they concluded that it was a novel force-generating protein and named it kinesin (from kinein, Greek for "to move").

Subsequent work focused both on characterizing the structure of kinesin as consisting of two globular heads and tail structures which bound to cargo and showing that the heads hydrolyzed ATP only when bound to microtubules (Scholey et al., 1989). Howard et al. (1989) developed an important technique, the single-molecule motility assay, through which they showed that a single kinesin molecule can produce movement and suggested that it moves along the microtubule by "coordinating" the activity of its heads such that one head releases at a time and moves past the 
Fig. 2 Schnapp et al., 1990 proposed that each kinesin head has a nucleotide-binding site and that each head alternately binds ATP. Copyright (1990) National Academy of Sciences

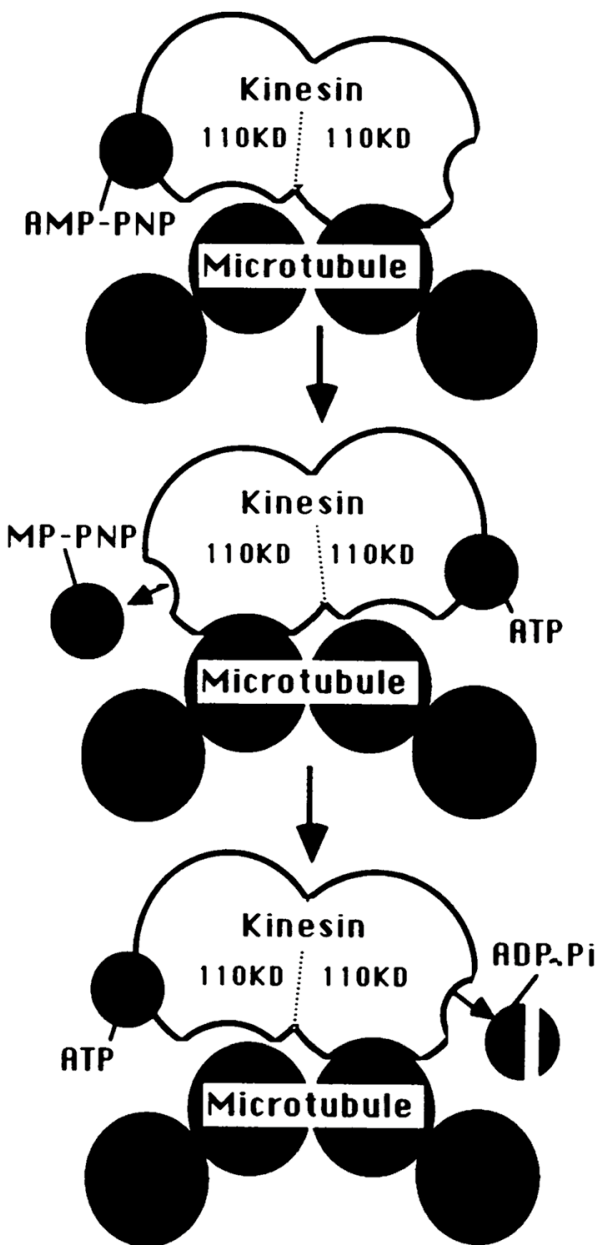

other thus, walking in a "hand-over-hand" fashion (Bollhagen 2021). Schnapp et al. (1990) proposed that this coordination was achieved by the binding of one head to ATP. This causes a conformational change in the other head, allowing it to bind to the microtubule and release nucleotide. The hydrolysis cycle thus "coordinates" the activity of the heads to ensure that one remains attached to the microtubule at all times (Fig. 2). Research in the following decade demonstrated that kinesin heads moved forward in discrete steps that correspond to the $8 \mathrm{~nm}$ repeats of tubulin (Svoboda et al., 1993) and that just one ATP was hydrolyzed per step (Coy et al., 1999; Hua et al., 1997; Schnitzer \& Block, 1997).

Further developing the account of how the steps of ATP hydrolysis map onto the movement of kinesin's two heads required use of more traditional biochemical techniques. Hackney (1994) found that when two-headed kinesin molecules with both heads bound to ADP associate with microtubules in the absence of ATP, only $50 \%$ of the ADP is released, whereas in the presence of ATP, $100 \%$ of the ADP 
is released. Further, $100 \%$ of ADP is released when a single headed kinesin construct binds to a microtubule in the absence of ATP. These findings suggested that when one head binds to a microtubule, it releases its ADP but that the other head is prevented from binding to the microtubule until the first head binds another ATP. Hancock and Howard (1999) further elaborated on the scheme of coordination by comparing the normal two-headed kinesin with a heterodimeric one-headed kinesin, showing that without the second head the kinesin detached an order of magnitude slower. From this they claimed that in the normal kinesin an internal strain between the heads serves to coordinate them, with the head bound to ADP being affected by the activity of the other head in expelling ADP, binding ATP, and hydrolyzing it (Fig. 3).

\subsection{Explaining motor movement in terms of the activities of ATP hydrolysis}

In this section, we have discussed how researchers developed mechanistic explanations for the phenomena of muscle contraction and intracellular transport. Researchers identified the responsible motors-myosin and kinesin, respectively-and decomposed the motors into entities and activities. The molecules' heads (entities) hydrolyze ATP (activities) which leads to the stepping (activity) of the heads. This is expressed economically in the diagrams which map the stages of the heads' hydrolytic cycles onto the stages of their mechanical steps. These mappings constitute a specification of the mechanisms for muscle contraction and intracellular transport in terms of the relevant entities and activities.

By certain philosophical standards, this is a remarkably complete explanation. Not only have the relevant entities and activities been identified but, further, these mappings establish relations of counterfactual dependence between stages of hydrolysis and stages of mechanical motion. Consider, for instance, step 1 of the Lymn-Taylor cycle depicted in Fig. 1. The diagram supports the following claims: (1) If the myosin head binds ATP (activity), the molecule releases the actin filament (activity). (2) If it does not bind ATP at that stage, the motor does not release from the actin filament. Likewise, the occurrence of each other stage in myosin's mechanical movement counterfactually depends upon the occurrence of the corresponding stage in the molecule's hydrolytic cycle. Arguably, this licenses a causal claim-the relevant stages in the molecule's hydrolytic activities cause the corresponding stages in their mechanical steps.

One might also think about the achievement these mappings represent in the following way. The representations of the stages of the molecules' mechanical steps (without the steps of ATP hydrolysis mapped onto it) constitute descriptions of the mechanisms merely in terms of state-transitions. As it stands, this is explanatorily inadequate by standard mechanist lights, "we think state transitions have to be more completely described in terms of the activities of the entities and how those activities produce changes that constitute the next stage (Machamer et al., 2000, p. 5). The concern here is that mechanisms are productive and a representation of a mechanism in terms of mere state-transitions fails to capture the productivity essential to understanding how a mechanism 


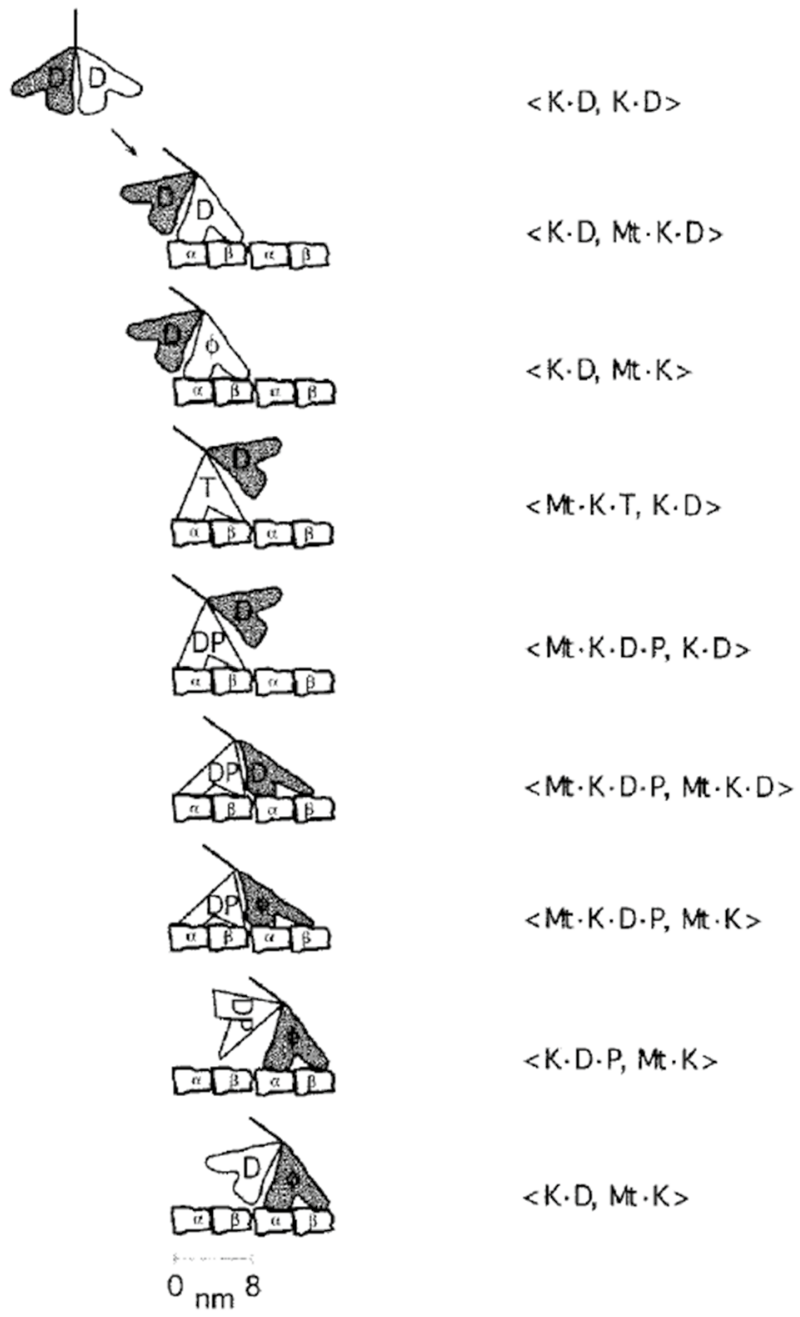

Fig. 3 Hancock and Howard (1999)'s model for the kinesin chemomechanical cycle. Copyright (1999) National Academy of Sciences

generates a phenomenon. By superimposing the stages of ATP hydrolysis onto the representations of the molecule's mechanical steps, we can see that the stages in the molecules' hydrolytic activities are the activities that advance the motors through their state transitions. Arguably, then, the mapping captures just that element of productivity that the standard approach to mechanistic explanation requires. Who could ask for anything more? Well, as we will see in the next section, biologists themselves. 


\section{Explaining the activities of molecular motor movement}

The research described in the previous section showed how the source of free energy, ATP, is coupled to the stepping activity of the myosin and kinesin motors by mapping stages in ATP binding, hydrolysis, and release of the products to stages of myosin's and kinesin's mechanical stepping. By standard mechanist lights, these constitute mechanistic explanations for the phenomena of muscle contraction and intracellular transport. As we will see in this section, however, researchers went further, and sought accounts of the means by which the molecules' hydrolytic activity causes the molecules to step. With its adherence to a fundamental dualism of entities and activities, the standard account of mechanistic explanation predicts that these accounts will be given in terms of a mechanistic explanation at a lower explanatory level that likewise specifies further entities and activities. This was not the case, however. Rather, researchers came to understand the molecules as themselves constituting characteristic sets of constraints on the free energy released in the course of ATP hydrolysis. In the constrained environment of the molecules, the chemical energy stored in ATP is transformed into mechanical energy to realize the stepping activities of the motors.

\subsection{Explaining the activity of myosin}

The first representation of the structure of the ATP binding site in myosin was created by Rayment et al. (1993) based on protein crystallography ${ }^{5}$ of chicken skeletal muscle (Fig. 4). Since this could be fitted to EM reconstructions of "decorated actin" produced by incubating actin filaments with isolated crossbridges without ATP, the researchers interpreted the image as showing the rigor state after ADP and Pi had been expelled and with myosin attached to actin (upper right corner in Fig. 1a and b). What this image showed was that the ATP binding site (labeled Nucleotide binding site) is at the opposite end of a $\beta$-sheet from the actin binding region, which is situated at the end of a cleft between the Lower $50 \mathrm{~K}$ and the Upper $50 \mathrm{~K}$ domains. Significantly, these sites are separated by about $40-50 \AA$. As a result, researchers concluded that the effects of the respective binding to actin or nucleotides must be communicated mechanically to the other site by physical changes in the $\beta$-sheet. The images also revealed a long helical tail, consisting of an $\alpha$ helix, that has the appearance of a lever arm. This tail is rigidly attached to the converter domain, suggesting that hydrolysis of ATP results in movement of the converter and the attached lever arm.

Individual crystal structure images are static and so do not reveal movements. To figure out the specific physical movement generated by hydrolysis that then resulted in movement along actin, researchers needed to compare this image with ones generated in other states. A few years later, several researchers (Fisher et al.,

\footnotetext{
5 The crystal structure for actin has been generated a few years earlier by Holmes et al. (1990). Actin was generally viewed as a passive component in muscle contraction, although it is now recognized to play crucial regulatory roles in the behavior of myosin.
} 


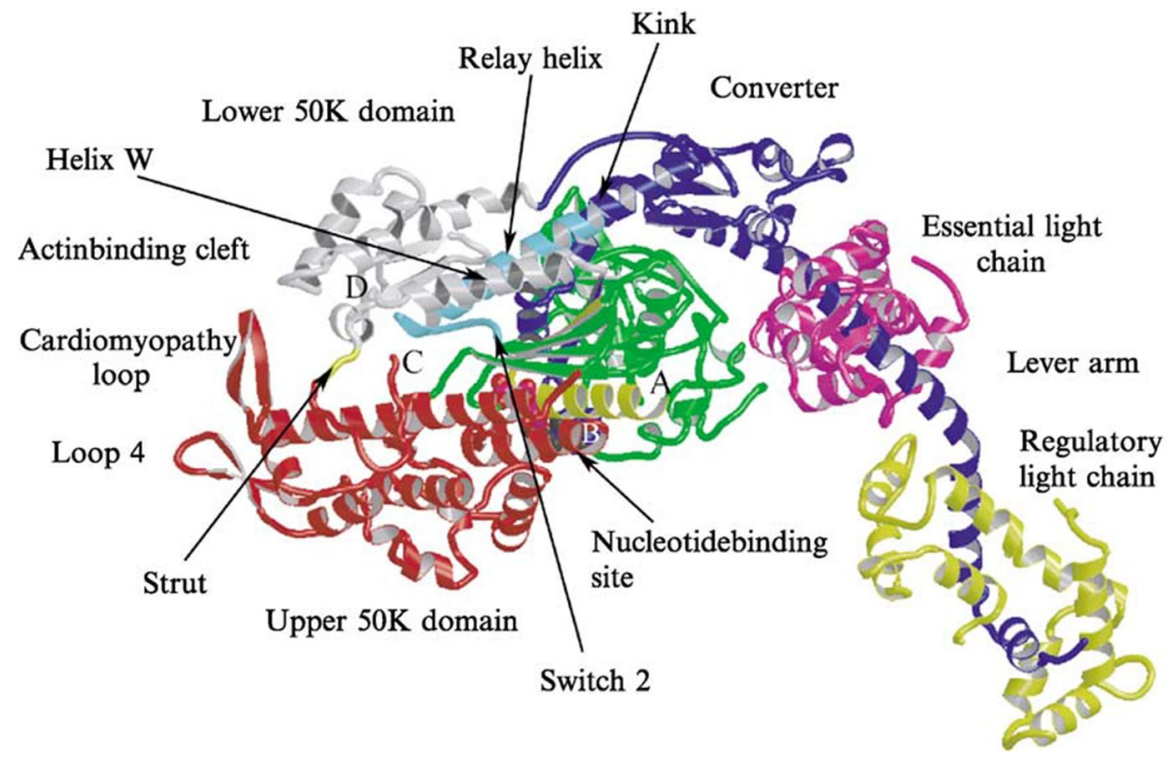

Fig. 4 Ribbon diagram of myosin motor domain in post-rigor state based on Rayment et al. (1993). Nucleotide binding site is shown in yellow. Actin binds in the cleft between the Upper $50 \mathrm{~K}$ domain and the Lower $50 \mathrm{~K}$ domain. The lever arm is in the post-powerstroke or down position. Reprinted from Advances in Protein Chemistry, Vol. 71, Geeves, M. A. \& Holmes, K. C., The molecular mechanism of muscle contraction, Fig. 2, Copyright (2005), with permission from Elsevier.

1995; Smith \& Rayment, 1995, 1996) generated crystal structures of myosin bound to molecules that bind as ATP does but do not undergo hydrolysis. These crystal structures were interpreted as presenting the pre-powerstroke whereas the first image represented the post-powerstroke state. Comparing the images revealed that the converter and lever arm had shifted by $60-70^{\circ}$ in the powerstroke. This angular difference was proposed to correspond to the $10 \mathrm{~nm}$ movement imposed on actin (see Holmes, 1996, 1997, for reviews). This provided an account of how the force generated in hydrolysis was transmitted to other parts of the myosin molecule.

Crystal structure images also revealed the structure of the ATP binding siteit was seen to involve a P-loop motif and switch 1 and 2 segments, very similar to those found in G-proteins. (Only switch 2 is shown in Fig. 4; switch 1 is in the region between the areas marked $\mathrm{A}$ and $\mathrm{B}$.) The comparison of the pre-powerstroke and rigor-like state shows that switch 2 moves $5 \AA$ : it starts in the closed state in contact with the $\gamma$-phosphate $(\mathrm{Pi})$ and enters the open state when it moves away. As in G-proteins, the closing of switch 2 involves the formation of an amide hydrogen bond between a glycine and the $\gamma$-phosphate of the nucleotide as part of the mechanism of hydrolysis. The formation of this bond also forces the Lower $50 \mathrm{~K}$ domain to rotate. This in turn forces movement in the attached relay helix (so named since it represents the communication pathway between the nucleotide binding site and the converter domain). Since the relay helix is forced up against a $\beta$-sheet, the attempted movement generates a kink that rotates the converter and lever arm $70^{\circ}$ (Holmes, 
2008; Smith \& Rayment, 1996). This tension stores the energy that will be released in the powerstroke.

Research over the subsequent two decades has led to important revisions and additions to this account, ${ }^{6}$ but they only reinforce the general picture that the chemical energy released in ATP hydrolysis is transformed into physical movement in the ATP binding site, which is then communicated to other parts of the myosin motor, changing its shape and hence its action on actin. This is illustrated in a recent model developed to accommodate evidence, based on FRET (fluorescence resonance energy transfer), that shows that ADP and Pi are released in different steps with both occurring after the powerstroke (Muretta et al., 2013, 2015). To fit the currently available data, Houdusse and Sweeney (2016) have advanced the scheme shown in Fig. 5. On their scheme, the powerstroke through which the myosin exerts force to pull itself along actin occurs after ATP hydrolysis and before the release of ADP and Pi. During the powerstroke the force built up in the abnormal position of the lever arm is released, and the lever arm moves $90^{\circ}$, back to its default position. Since the myosin is bound to actin at this time, the effect is to pull the rest of the myosin filament along the actin filament. Once the ADP and Pi are expelled, myosin detaches from actin and prepares for another powerstroke by binding a new ATP. This conformation change at the binding site results in re-cocking the lever arm. Since myosin is not attached to actin, the myosin head moves freely and is positioned further along the actin. Little free energy is released at this step. With hydrolysis and the subsequent binding of myosin at the new position on actin, force is built up, ready to be released with a new powerstroke. Thus, the free energy released in ATP hydrolysis is temporarily constrained to maintain the lever arm in its position until, in the next powerstroke, it is released.

Although research is ongoing and will likely result in further modifications of this account, it provides a model of how release of free energy is constrained to generate the activity of the myosin motor. The research reveals that muscle contraction or even the cycle of myosin engagements with actin are not primitive activities, but ones that can be explained in terms of the constraints on the free energy released in the hydrolysis of ATP.

\footnotetext{
${ }^{6}$ For example, images by Coureux et al. (2003), Holmes et al. (2004), and Holmes et al. (2003) showed that when myosin is the rigor state, bound to actin without the nucleotide, the cleft between the Upper $50 \mathrm{~K}$ and Lower $50 \mathrm{~K}$ domains is closed. Drawing on these, Coureux et al. (2004) advanced an interpretation according to which binding to actin rotated the Lower $50 \mathrm{~K}$ domain and resulted in the opening of the nucleotide binding site. When a new ATP molecule is bound into the nucleotide site, the rotation of the Lower $50 \mathrm{~K}$ domain is reversed, reopening the actin binding site. Binding a new ATP also alters the conformation of the P-loop and switches 1 and 2 so as to favor ATP hydrolysis. This further supported the interpretation that the opening of Switch 2 rotates the -sheet and creates a kink in the relay helix that moves the converter, creating mechanical stress which is released in the power stroke and causes the lever arm to rotate back.
} 


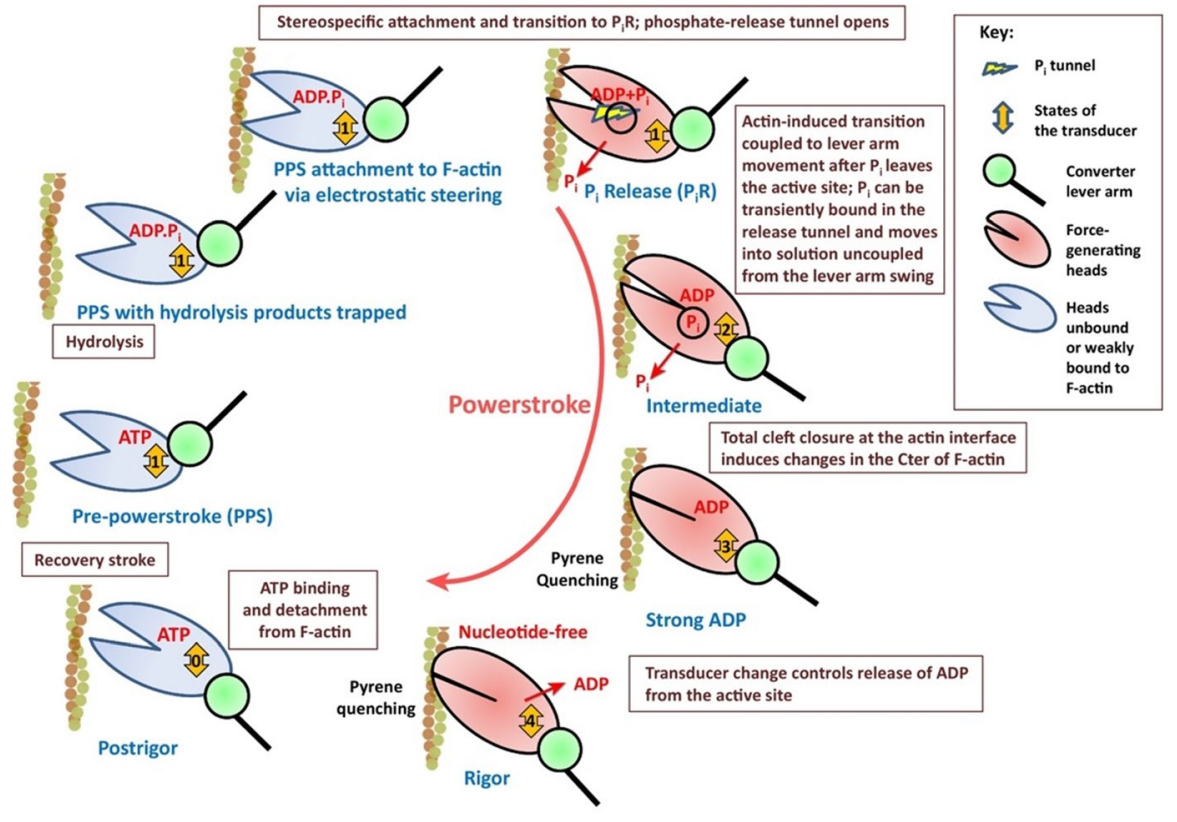

Fig. 5 A current cartoon of how ATP hydrolysis, shown on the left, generates forces that are then released in the powerstroke shown on the right. Reprinted from Trends in Biochemical Sciences, 41, Houdusse, A. \& Sweeney, H. L., How myosin generates force on actin filaments, Fig. 1, Copyright (2016), with permission from Elsevier

\subsection{Explaining the activity of kinesins}

As with myosins, explaining the activity of kinesins required understanding how chemical energy from ATP is translated into mechanical energy in the kinesin molecule, resulting in this case, in the movement of one kinesin head in front of the other. After the discovery and initial characterization of kinesin, researchers assumed it was unlikely that it would operate in the same manner as myosin. First, kinesin is about half the size of myosin. Second, while a myosin generates a single powerstroke while attached to actin and then dissociates, an individual kinesin takes on the order of 100 steps along a microtubule before dissociating. Third, initial genetic sequencing of the two molecules did not suggest any homologies between the molecules. The research that ensued, however, resulted in a remarkably similar account of kinesin movement, one involving the constrained release of chemical energy from ATP, resulting in characteristic changes in kinesin's conformation which constitute its stepping activity.

The first step in working out the mechanism of kinesin movement was taken when Kull et al. (1996) identified the crystal structure of kinesin bound to ADP and showed it to be similar to that which Rayment had identified for myosin just a couple years earlier. Using the three-dimensional structure as a guide, researchers then discovered homologous sequences in myosin and kinesin (Kull et al., 1998; 


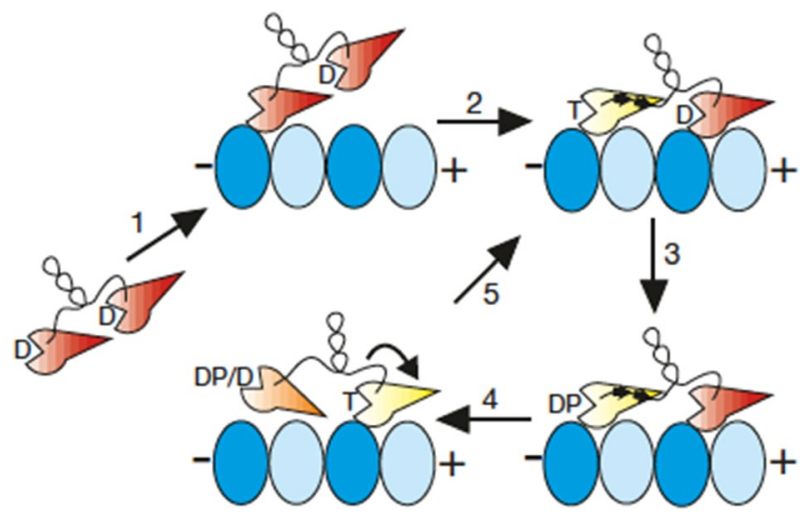

Fig. 6 Changes in linker docking as a kinesin walks. Dark and light blue ovals indicate successive $\alpha$ and $\beta$ subunits of tubulin. Kinesin in the state bound to ATP and docked on microtubule is shown in yellow; in other states it is shown in red. D indicates binding of the kinesin to ADP, T to ATP, and DP to ADP and Pi. Black dots indicate docking of linker to catalytic core of the kinesin, which results in generating force that moves the other head forward. From Rice et al. (1999). Reprinted by permission from Nature, A structural change in the kinesin motor protein that drives motility, Rice et al. (1999)

Vale \& Milligan, 2000), which provided insight into the parts of the kinesin molecule involved in binding the $\gamma$-phosphate of the nucleotide.

The activity of kinesin in response to release of free energy is different from that of myosin, and this requires explanation. Research showed that it is due to differences in the way in which energy release is constrained. Kinesin does not have the elongated lever arm found in myosin. Instead, each head is connected to a neck linker which then connects via a common coiled-coil to cargo (Kozielski et al., 1997). Comparing crystal structures of kinesin bound to ADP in rat (Kozielski et al., 1997) and human (Kull et al., 1996) revealed a difference in the position of the neck linker. Rather than assuming that it was a species difference, researchers inferred a difference in the state of the motor molecule when it was crystalized. Moreover, they hypothesized that the neck linker played a similar role to the lever arm in myosin-its movement resulted from the way in which free energy released in ATP hydrolysis exerts forces that alter the conformation of the molecule.

Unable, at the time, to solve the crystal structure of kinesin in different states of ATP hydrolysis, Rice et al. (1999) instead used spectral analysis to show different conformations of the neck linker when kinesins are in different nucleotide and microtubule binding states. These researchers concluded that the neck linker is generally in an unstructured and so flexible state and becomes more ordered and immobile when the microtubule bound kinesin binds ATP (see step 5 in Fig. 6 in which the position of the linker has changed to pointing forward from the head that is now in the rear). As a result of being in this structured state, the kinesin head exercises a force on the other head, moving it forward. With fluorescence resonance energy transfer (FRET), they measured the distance between specific residues and concluded that the neck linker docks onto the catalytic core of the kinesin, a proposal they further supported with cryo-EM. 

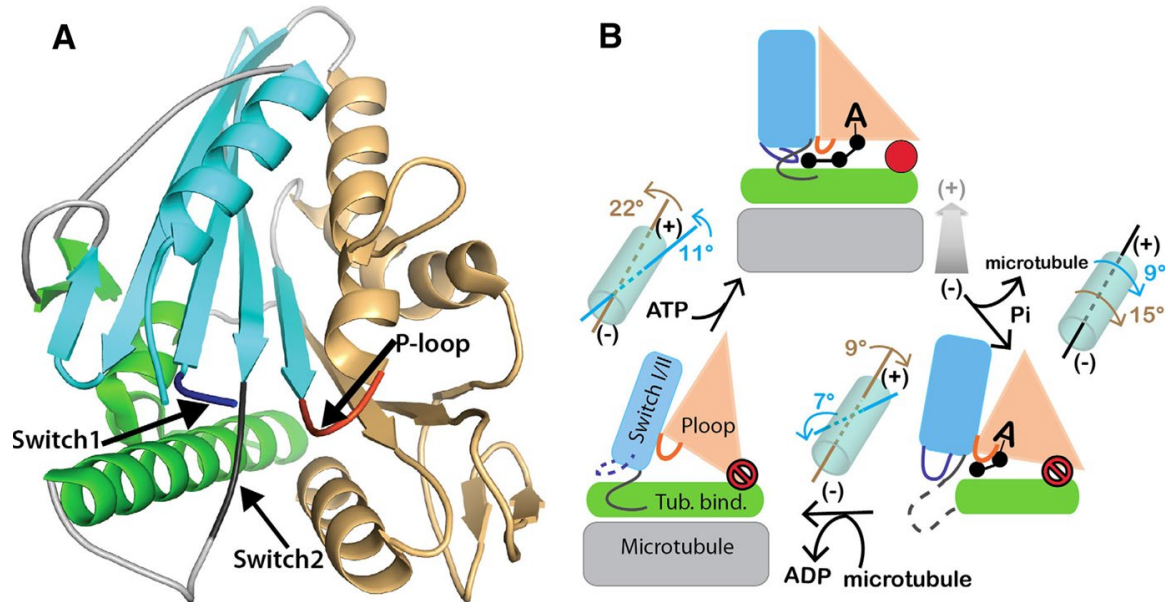

Fig. 7 a Ribbon diagram of kinesin showing the location of switches 1 and 2 and the p-loop. b A cartoon of a proposed mechanism by which the movements generated by ATP binding and hydrolysis open and close a locus for neck linker docking. Reprinted from Protein Science, 24, Wang, W. et al., Kinesin, 30 years later: Recent insights from structural studies, Fig. 2, Copyright (2015), with permission from Wiley

In the last decade, with the development of high-resolution X-ray crystallography (resolution to $2.2 \AA$ ) and cryo-electron microscopy (resolution to $6 \AA$ ), researchers obtained images of both unbound kinesin and kinesin bound to tubulin and ATP or ADP that provided a clear image of how energy was transformed in kinesins. Comparing images of kinesin in different states, Cao et al. (2014) concluded that "the kinesin structural changes along the nucleotide cycle are well described by rigidbody movements of three motor subdomains": the switch 1/2 subdomain, the P-loop subdomain, and the tubulin binding subdomain. In particular, the crystal structures reveal that when ATP binds to the P-loop, as shown by the A with three connected back circles in the top portion of Fig. 7b, the P-loop (orange triangle) and switches 1 and 2 (blue rectangle) align so that a place is opened for the neck-linker (red circle) to dock. This is due in large part to the fact that the first residue of the P-loop, an isoleucine, gets buried in the cavity. This creates the configuration that catalyzes ATP hydrolysis. The hydrolysis and expulsion of Pi (while still bound to ATP) exerts a force that causes the P-loop and two switches to mechanically reconfigure, resulting in the closure of the docking site and the kinesin detaching from the microtubule. While detached, the docking of the other head thrusts this head forward. Once it expels ADP, this head can again bind to the microtubule, now at a location further along the microtubule. Although this changes the conformation of the P-loop and the switches, the neck-linker docking site remains blocked. It only opens again when a new molecule of ATP is bound (Wang et al., 2015).

As in the case of myosin, research is ongoing and, while supporting the general picture of how energy from ATP hydrolysis is constrained to force mechanism movement, has challenged the details. According to Sindelar and Liu (2017), new research by Milic et al. (2014) and Mickolajczyk et al. (2015) is generating "A quiet 
revolution in the kinesin field [that] has recently contradicted the longstanding idea that the forward step by one-head-bound kinesin is triggered by ATP binding, establishing that the forward step instead occurs after hydrolysis of ATP." This led Hancock (2016), to advance a new consensus model according to which binding to ATP by the forward head enhances the release of the trailing head from the microtubule while hydrolysis results in docking of the linker and the forward movement of the previously trailing head.

Although this recent research has altered the details, the basic categories in terms of which the activity of kinesin motors are explained remains the same: the constrained hydrolysis of ATP results in the free energy released exercising force that alters the conformation in both heads of the kinesin motor, resulting in the activity of walking along microtubules.

\subsection{Explaining the activity of motor movement}

The research on both kinesin and myosin discussed in this section resulted in accounts that explain their activities. In both cases, researchers showed how the free energy released in ATP hydrolysis results in conformational changes to the binding pocket. These changes in turn apply forces to other parts of the motor, including the site of actin or microtubule binding. The changes at the binding site are responsible for the cycle of binding and unbinding from actin or the microtubules. The conformation changes generated by hydrolysis at the ATP binding site are also communicated to other parts of the molecule. In myosin they build up tension that forces a rotation of the level arm, which is then released in the powerstroke that pulls myosin along the attached actin. In the case of kinesin, force is applied to the neck linker that then serves to move the rear head in front where it finds a new binding site. Thus, both motors generate motion as a result of ATP hydrolysis in a constrained pocket creating forces that alter other parts of the motors. The differences in the activity of the two motors result from the different constraints imposed by the structural differences in the other parts of the molecules.

\section{Explaining activities in biological mechanisms}

On the standard account of mechanistic explanation, scientists explain a phenomenon by appealing to the entities and activities that constitute it. If they desire to explain the activities of one of the component entities, they repeat the process. Science that has followed this procedure has been enormously productive. Nothing in this paper is intended to downplay the contributions of such mechanistic research or philosophical accounts of it. (Below, though, we argue that an important addition to such accounts is to identify the source of free energy on which activities depend). Yet, at some point, such research typically bottoms out. As the new mechanists have discussed, this often occurs when researchers lack the tools or the interest in explaining the activities of the components they have identified. These are simply accepted. This process leaves the active nature of activities unexplained. Dualists 
Fig. 8 Illustration of A. unconstrained object subject to three degrees of freedom and B. a constrained object, limited to one degree of freedom. From https://www.cs.cmu.edu/ rapid proto/mechanisms/chpt $4 . h$ tml
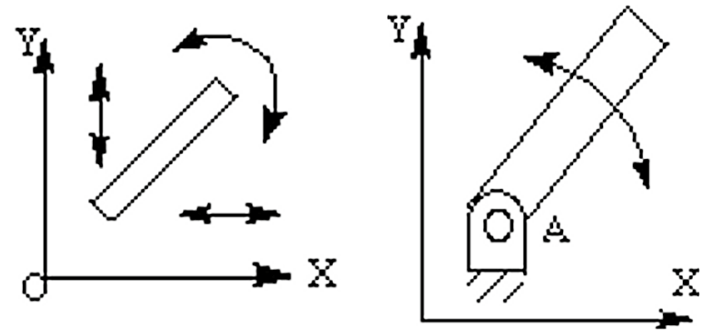

such as MDC are happy to leave matters there. This is unsatisfying, however, if one wishes to understand how mechanisms are active.

The research we have discussed in the previous section identifies a way in which biologists have gone further and explained activities of mechanisms in more fundamental terms. The first step is to recognize that mechanisms are only active when free energy is employed in them. This requirement has not been emphasized in the accounts of the new mechanists, but it is fundamental. A mechanism without free energy will not perform work. Any movement will be due to external forces impinging on them or to thermal noise (Brownian motion). When there is a source of free energy in the mechanism, it can generate activity. Once one focuses on the need for free energy for a mechanism to perform activities, one can take the second step. What happens to the free energy depends on how it is constrained. If not constrained, it simply dissipates, and entropy increases, without work being performed. When it is constrained, work can be performed, with the nature of that work depending on the constraints imposed. As illustrated in the case of molecular motors, constraints are provided by the physical parts of the mechanism and the way they are organized. As a result of these organized parts, free energy release is constrained in a particular manner, giving rise to the activities of the different parts.

As we have shown, mechanistic explanations of molecular motors appeal to energy and to the role parts of mechanisms play in constraining its release. We thus advocate for incorporating these into the mechanistic framework. The fundamental roles each performs is illustrated by a simple example from classical mechanics. At the core of Newtonian physics is the idea of force acting on objects. Consider the rectangular object shown in the $2 \mathrm{D}$ space on the left of Fig. 8. It has 3 degrees of freedom: it can move along the $\mathrm{X}$ and $\mathrm{Y}$ axes and can rotate. If a force is applied to it, it can move in any of these directions. The same object is shown on the right connected to a hinge. The hinge is a constraint on the movement of the rectangle: it reduces the object's degrees of freedom. Now it can only rotate when a force is applied to it. As articulated by Hooker (2013), constraints are both limiting (the rectangle can no longer move along the $\mathrm{x}$ axis) and enabling (it would have been difficult to apply a force that would get the rectangle to rotate counterclockwise and not along the $\mathrm{x}$ or $\mathrm{y}$-axis in the situation on the left. When the rectangle is constrained by the hinge it is relatively easy: simply apply a force anywhere along the bottom edge of the rectangle.

The forces appealed to in Newtonian physics result from free energy. Imagine that the angle labeled A in Fig. 8 is a nucleotide binding pocket. If ATP hydrolysis 
occurs in that pocket, the chemical energy stored in the ATP molecule is translated into a mechanical force applied to the rectangle. This shows up as an activity-rotating. What we have here, then, is an entity engaging in an activity. But, further, we have an account of the means by which the rectangular object rotates-an account in terms of energetics and constraints. We draw this same lesson from the case of molecular motors research. Figure 8 presents in a very simplified form the basic explanatory principles appealed to in the explanations of the molecular motors and illustrated in Figs. 4, 5, and 7). The chemical energy released in the nucleotide binding pocket in each motor is translated into mechanical forces which alter the shape of the molecule. The particular constraints result in movements that constitute the activity of stepping. Given the greater number of parts acting as constraints, the kinematic activity in the molecular motors is in obvious ways much more complex than what is represented in Fig. 8. Nonetheless, the basic explanatory principles are the same-energetics and constraints.

One might worry that in appealing to free energy to explain activities, we have replaced a dualism of entities and activities with one of constraints and free energy. In one sense, we have: both free energy and constraints are required in the explanation of an activity. But the need for free energy is well-recognized in physics. The principles of thermodynamics are central to understanding what happens in the universe. A dualism of matter and energy is forced on us by physics. As a "descriptive ontology of science" (Illari and Williamson 2013), such a dualism is well motivated. The category of activities, however, is largely a philosophical invention. When MDC introduce activities, they appeal to examples ("fitting, turning, opening, colliding, bending and pushing...") to illustrate them. Moreover, these activities prompt questions: Why does this entity turn or open? What enables one entity to push another? Invoking activities as a distinct ontological category does not advance us towards an explanation of these activities - it simply reifies the fact that mechanistic components are active. The different ways in which free energy is constrained provide answers to these questions in terms of a category already required to explain everything that happens in the physical universe.

To be clear, however, we are not arguing for the elimination of activities as an analytic category in terms of which to explicate the structure of explanation in the biological sciences. Further, we are not arguing that mechanistic explanations that appeal to activities and do not explain them in terms of free energy are therefore explanatorily deficient. Entities in mechanisms do engage in activities and characterizing these activities allows one to explain how a mechanism in which these activities occur gives rise to a phenomenon. We are simply showing that activities are subject to further analysis and this analysis dispels the metaphysical mystery that surrounds activities when they are characterized as ontologically fundamental.

On the traditional account, activities in a mechanism at one level are explained in terms of the entities and activities of a mechanism at a lower explanatory level. When researchers explain activities in terms of energetics and constraints, are they likewise doing so at a lower explanatory level? We think not. The entities into which the molecules are decomposed (e.g., nucleotide binding site, actin or microtubule binding site, neck linker, lever arm) and the activities ascribed to each of them (e.g., binding and releasing nucleotides, actin, or the microtubule, moving the lever arm 
or the neck linker) are not further decomposed into more basic entities performing more basic activities. Rather, for the purpose of explaining the activities of these entities, the entities themselves are construed as a set of constraints that respond in characteristic ways as free energy flows through them. The forces applied to the binding sites or to the linker, etc., just are the constrained release of free energy. What one has done is redescribed basic activities in terms of constrained release of free energy and thereby explained why they are active.

We have focused our case on molecular motors, and one might question whether the account of activities in molecular motors generalizes. We offer two reasons to think they are not an exceptional case. First, in addition to contraction of muscles and transport along microtubules, molecular motors perform many other activities in cells. Dyneins, for example, figure in both the movement of cilia and in positioning of chromosomes during cell division. The synthesis of ATP in the mitochondrion relies on the $\mathrm{F}_{0} \mathrm{~F}_{1}$-ATP synthase molecular motor operating in reverse-using the free energy released from a proton gradient to turn a rotor that positions ADP and Pi appropriately to form ATP. The synthesis of microtubules depends on a molecular motor that uses GTP rather than ATP. Numerous nucleic acid motors, including RNA polymerase, DNA polymerase, and the ribosome, figure in gene expression. Far from being an unusual biological mechanism, biological motors constitute a large category of biological mechanisms responsible for many activities of cells.

Second, a host of other cell mechanisms that are not generally classed as motors but that are critical to the functioning of cells rely on ATP or GTP as their source of free energy. For example, maintenance of appropriate concentrations of ion and proton gradients across membranes is critical for a variety of cell activities. These rely on pumps or transporters that utilize ATP or GTP to move ions or protons across the cell membrane against a concentration gradient. Perhaps the best known of these is the sodium-potassium pump that transports three sodium ions out of the cell and two potassium ions into the cell. In this case, binding and hydrolysis of ATP phosphorylates the pump protein, altering the conformation of the molecule so as to release sodium ions outside the cell and binding potassium ions. Binding potassium reverses the conformation change, resulting in release of potassium inside the cell and again binding sodium.

We have pitched our account of how to explain activities within mechanisms at the foundational level at which mechanistic explanations in biology bottom out. It is at this level where the chemical energy produced in metabolism gets translated into mechanical energy. The research on molecular motors we have discussed demonstrates directly how to explain activities in terms of energetics and constraints. However, energetics and constraints are also relevant to understanding mechanisms at higher levels of organization-ones traditionally explicated in terms of entities and activities. Even if not overtly explained in terms of energetics and constraints, ultimately, at any level, the activities of a mechanism are the result of free energy being constrained to perform work. Accounts of how this occurs in specific mechanisms will vary from mechanism to mechanism but, across all cases, energetics and constraints provide the fundamental terms in which the mechanism's activities as such can be understood. In short, bringing energetics and constraints into the mechanistic fold vindicates, philosophically, 
the appeal to activities even in mechanistic explanations that do not explicitly cash out their appeals to activities in energetic terms. To use an analogy, just as, some argue, Darwin legitimized talk of design in biology by showing how it can be brought about by means that pass naturalistic muster, we claim to have legitimized talk of mechanistic activities by showing how they can be analyzed in terms of energetics and constraints.

What does it look like to explain the activities of mechanistic entities at levels of organization higher than the bottom-out level in energetic terms? This involves identifying the point at which free energy enters the system at that level. We illustrate this by returning to the case of muscle movement resulting from ATP hydrolysis by myosin. The work performed by the contraction of muscle cells depends on the specific tendons that attach muscles to bones and ligaments that attach bones to one another. These further constrain the energy released in the action of the motor. The ability of myosin to bind to actin depends upon release of $\mathrm{Ca}^{++}$ from the sarcoplasmic reticulum in response to action potentials generated in muscles as a result of the action potentials in nerves reaching the neural muscular junction. These in turn depend upon activity in the central nervous system that, in part, depends on events in the organism's environment. Both of these require further sources of free energy made available by pumps that create the $\mathrm{Ca}^{++}$gradient across the sarcoplasmic membrane and pumps that create ion gradients over the membranes of neurons. Emphasizing the role of the constrained release of free energy in explaining activities applies across all levels in the mechanistic hierarchy. Higher-level activities, just as those at the bottom-out level, depend upon the release of energy. Higher-level entities also constrain those at the bottom level, determining how energy released in molecular motors, ion pumps, etc. results in activities at higher levels.

As we mentioned, explanations of the activities of higher levels can, especially in light of our philosophical vindication of the category of activities, reference activities at the next lower level without themselves revealing how free energy gives rise to those activities. Accordingly, mechanistic explanation above the foundational level where the constrained release of free energy results in movement can be conducted in the standard manner characterized by the new mechanists. Yet, we contend that even these accounts would benefit from attending to where free energy is supplied to these mechanisms. On the one hand, it will help localize where the work is performed that explains the activity of the mechanism. On the other, it will provide a reference point for understanding the operation of the mechanism. Machamer et al. (2000) present mechanisms as operating from "start or set-up to finish or termination conditions" (p. 3) Given the cyclic organization found in many biological mechanisms, one can question whether there are principled start or termination conditions. The entry of free energy provides a principled starting point for analyzing the activity in the mechanism and the products of the work performed by the constrained release of energy provides a plausible set of termination conditions. Moreover, even if one does not directly track energy transduction in developing one's account of the mechanism, one can plausibly infer that these activities result from the constrained flow of free energy through the mechanism and so organize the account of the mechanism around that flow. 


\section{Conclusion}

The components of mechanisms are active. The standard account of mechanistic explanation explains activities in terms of activities and does not offer an explanation other than to appeal to other activities. To illustrate how scientists in fact provide explanations of activity without appeal to other activities, we have focused on two molecular motors, myosins and kinesins. The research on both motors that we analyzed in Sect. 2 proceeded in the manner characterized by the new mechanists, ultimately showing how stages in ATP hydrolysis corresponded to steps in the stepping behavior of both motors. But, as we showed in Sect. 3, research on molecular motors has taken an additional step, showing where free energy is released from hydrolysis of ATP and how it leads to conformation changes in other parts of the molecule that constitute the stepping. The motor provides a set of constraints in which free energy is directed into forces that generate movements that corresponds to the activities of the motor. In Sect. 4 we flesh out the framework advanced in our analysis-appealing to free energy and its constrained release to explain activities. As a result of the constrained release of free energy, biological mechanisms are active, but their activities are not primitive posits. They can be explained.

Acknowledgements This paper developed out of the Active Matter Workshop held in the summer of 2018 at Georgetown University that was supported by the John Templeton Foundation. We are especially thankful for the efforts of Pranab Das and Patrick McGivern in organizing this workshop. We also extend special thanks also to Jason Winning for very helpful feedback on early drafts of the manuscript.

Open Access This article is licensed under a Creative Commons Attribution 4.0 International License, which permits use, sharing, adaptation, distribution and reproduction in any medium or format, as long as you give appropriate credit to the original author(s) and the source, provide a link to the Creative Commons licence, and indicate if changes were made. The images or other third party material in this article are included in the article's Creative Commons licence, unless indicated otherwise in a credit line to the material. If material is not included in the article's Creative Commons licence and your intended use is not permitted by statutory regulation or exceeds the permitted use, you will need to obtain permission directly from the copyright holder. To view a copy of this licence, visit http://creativecommons.org/licen ses/by/4.0/.

\section{References}

Adams, R. J. (1982). Organelle movement in axons depends on ATP. Nature, 297(5864), 327-329.

Allen, R. D., Allen, N. S., \& Travis, J. L. (1981). Video-enhanced contrast, differential interference contrast (AVEC-DIC) microscopy: A new method capable of analyzing microtubule-related motility in the reticulopodial network of Allogromia laticollaris. Cell Motility, 1(3), 291-302.

Allen, R. D., Metuzals, J., Tasaki, I., Brady, S. T., \& Gilbert, S. P. (1982). Fast axonal transport in squid giant axon. Science, 218(4577), 1127-1129.

Bechtel, W., \& Abrahamsen, A. (2005). Explanation: A mechanist alternative. Studies in History and Philosophy of Biological and Biomedical Sciences, 36(2), 421-441.

Bechtel, W., \& Richardson, R. C. (1993/2010). Discovering complexity: Decomposition and localization as strategies in scientific research. Cambridge, MA: MIT Press. 1993 edition published by Princeton University Press. 
Bollhagen, A. (2021). The inchworm episode: Reconstituting the phenomenon of kinesin motility. European Journal for Philosophy of Science. https://doi.org/10.1007/s13194-021-00358-5

Cao, L. Y., Wang, W. Y., Jiang, Q. Y., Wang, C. G., Knossow, M., \& Gigant, B. (2014). The structure of apo-kinesin bound to tubulin links the nucleotide cycle to movement. Nature Communications, 5 , 5364. https://doi.org/10.1038/ncomms6364

Cartwright, N. (1999). The dappled world: A study of the boundaries of science. Cambridge University Press.

Coureux, P. D., Sweeney, H. L., \& Houdusse, A. (2004). Three myosin V structures delineate essential features of chemo-mechanical transduction. EMBO Journal, 23(23), 4527-4537. https://doi.org/10. 1038/sj.emboj.7600458

Coureux, P. D., Wells, A. L., Menetry, J., Yengo, C. M., Morris, C. A., Sweeney, H. L., \& Houdusse, A. (2003). A structural state of the myosin V motor without bound nucleotide. Nature, 425(6956), 419-423. https://doi.org/10.1038/nature01927

Coy, D. L., Wagenbach, M., \& Howard, J. (1999). Kinesin takes one 8-nm step for each ATP that it hydrolyzes. Journal of Biological Chemistry, 274(6), 3667-3671.

Craver, C. F., \& Darden, L. (2013). In search of mechanisms: Discoveries across the life sciences. University of Chicago Press.

De Robertis, E., \& Franchi, C. M. (1953). The submicroscopic organization of axon material isolated from myelin nerve fibers. Journal of Experimental Medicine, 98(3), 269-276.

Engelhardt, W. A., \& Ljubimowa, M. N. (1939). Myosine and Adenosinetriphosphatase. Nature, 144, 668. https://doi.org/10.1038/144668b0

Fisher, A. J., Smith, C. A., Thoden, J., Smith, R., Sutoh, K., Holden, H. M., \& Rayment, I. (1995). Structural studies of myosin-nucleotide complexes: A revised model for the molecular basis of muscle contraction. Biophysical Journal, 68(4), S19-S28.

Glennan, S., \& Illari, P. M. (Eds.). (2018). The Routledge handbook of mechanisms and mechanical philosophy. Routledge.

Grafstein, B., \& Forman, D. S. (1980). Intracellular transport in neurons. Physiological Reviews, 60(4), 1167-1283. https://doi.org/10.1152/physrev.1980.60.4.1167

Hackney, D. D. (1994). Evidence for alternating head catalysis by kinesin during microtubule-stimulated ATP hydrolysis. Proceedings of the National Academy of Sciences United States of America, 91(15), 6865-6869.

Hancock, W. O. (2016). The kinesin-1 chemomechanical cycle: Stepping toward a consensus. Biophysical Journal, 110(6), 1216-1225. https://doi.org/10.1016/j.bpj.2016.02.025

Hancock, W. O., \& Howard, J. (1999). Kinesin's processivity results from mechanical and chemical coordination between the ATP hydrolysis cycles of the two motor domains. Proceedings of the National Academy of Sciences of the United States of America, 96(23), 13147-13152. https://doi.org/10. 1073/pnas.96.23.13147

Hitchcock-DeGregori, S. E., \& Irving, T. C. (2014). Hugh E. Huxley: The compleat biophysicist. The Biophysicist Journal, 107(7), 1493-1501. https://doi.org/10.1016/j.bpj.2014.07.069

Holmes, K. C. (1996). Muscle proteins: Their actions and interactions. Current Opinion in Structural Biology, 6(6), 781-789. https://doi.org/10.1016/S0959-440x(96)80008-X

Holmes, K. C. (1997). The swinging lever-arm hypothesis of muscle contraction. Current Biology, 7(2), R112-118.

Holmes, K. C. (2008). Myosin structure. In L. M. Coluccio (Ed.), Myosins: A Superfamily of Molecular Motors (pp. 35-54). Springer.

Holmes, K. C., Angert, I., Jon Kull, F., Jahn, W., \& Schröder, R. R. (2003). Electron cryo-microscopy shows how strong binding of myosin to actin releases nucleotide. Nature, 425(6956), 423-427. https://doi.org/10.1038/nature02005

Holmes, K. C., Popp, D., Gebhard, W., \& Kabsch, W. (1990). Atomic model of the actin filament. Nature, 347(6288), 44-49. https://doi.org/10.1038/347044a0

Holmes, K. C., Schroder, R. R., Sweeney, H. L., \& Houdusse, A. (2004). The structure of the rigor complex and its implications for the power stroke. Philosophical Transactions of the Royal Society of London Series B-Biological Sciences, 359(1452), 1819-1828. https://doi.org/10.1098/rstb.2004. 1566

Hooker, C. A. (2013). On the import of constraints in complex dynamical systems. Foundations of Science, 18(4), 757-780. https://doi.org/10.1007/s10699-012-9304-9

Houdusse, A., \& Sweeney, H. L. (2016). How myosin generates force on actin filaments. Trends in Biochemical Sciences, 41(12), 989-997. https://doi.org/10.1016/j.tibs.2016.09.006 
Howard, J., Hudspeth, A. J., \& Vale, R. D. (1989). Movement of microtubules by single kinesin molecules. Nature, 342(6246), 154-158.

Hua, W., Young, E. C., Fleming, M. L., \& Gelles, J. (1997). Coupling of kinesin steps to ATP hydrolysis. Nature, 388, 390. https://doi.org/10.1038/41118

Huxley, A. F. (1977). Looking back on muscle. In A. L. Hodgkin, A. F. Huxley, W. Feldberg, W. A. H. Rushton, R. A. Gregory, \& R. A. McCance (Eds.), The pursuit of nature: Informal essays on the history of physiology (pp. 23-64). Cambridge University Press.

Huxley, A. F., \& Niedergerke, R. (1954). Structural changes in muscle during contraction - Interference microscopy of living muscle fibres. Nature, 173(4412), 971-973.

Huxley, H. E. (1958). The contraction of muscle. Scientific American, 199, 66-82.

Huxley, H. E. (1969). The mechanism of muscular contraction. Science, 164(3886), 1356-1365.

Huxley, H. E., \& Hanson, J. (1954). Changes in the cross-striations of muscle during contraction and stretch and their structural interpretation. Nature, 173(4412), 973-976.

Illari, P. M., \& Williamson, J. (2013). In defence of activities. Journal for General Philosophy of Science, 44(1), 69-83.

Klein, C. (2018). Mechanisms, resources, and background conditions. Biology and Philosophy, 33(5), 36. https://doi.org/10.1007/s10539-018-9646-y

Kozielski, F., Sack, S., Marx, A., Thormahlen, M., Schonbrunn, E., Biou, V., \& Mandelkow, E. (1997). The crystal structure of dimeric kinesin and implications for microtubule-dependent motility. Cell, 91(7), 985-994.

Kühne, W. F. (1864). Untersuchungen über das Protoplasma und die Contractilitat. W. Engelmann.

Kull, F. J., Sablin, E. P., Lau, R., Fletterick, R. J., \& Vale, R. D. (1996). Crystal structure of the kinesin motor domain reveals a structural similarity to myosin. Nature, 380(6574), 550-555. https://doi.org/ $10.1038 / 380550 \mathrm{a} 0$

Kull, F. J., Vale, R. D., \& Fletterick, R. J. (1998). The case for a common ancestor: Kinesin and myosin motor proteins and G proteins. Journal of Muscle Research and Cell Motility, 19(8), 877-886. https://doi.org/10.1023/A:1005489907021

Lasek, R. J., \& Brady, S. T. (1984). Adenylyl imidodiphosphate (AMP-PNP), a non-hydrolyzable analogue of ATP produces a stable inter-mediate in the motility cycle of fast axonal transport. Biological Bulletin, 167, 503.

Lasek, R. J., \& Brady, S. T. (1985). Attachment of transported vesicles to microtubules in axoplasm is facilitated by AMP-PNP. Nature, 316(6029), 645-647. https://doi.org/10.1038/316645a0

Lymn, R. W., \& Taylor, E. W. (1971). Mechanism of adenosine triphosphate hydrolysis by actomyosin. Biochemistry, 10(25), 4617-4624. https://doi.org/10.1021/bi00801a004

Machamer, P. (2004). Activities and Causation: The Metaphysics and Epistemology of Mechanisms. International Studies in the Philosophy of Science, 18(1), 27-39. https://doi.org/10.1080/02698 590412331289242

Machamer, P., Darden, L., \& Craver, C. F. (2000). Thinking about mechanisms. Philosophy of Science, $67,1-25$.

Mickolajczyk, K. J., Deffenbaugh, N. C., Ortega Arroyo, J., Andrecka, J., Kukura, P., \& Hancock, W. O. (2015). Kinetics of nucleotide-dependent structural transitions in the kinesin-1 hydrolysis cycle. Proceedings of the National Academy of Sciences, 112(52), E7186. https://doi.org/10.1073/pnas. 1517638112

Milic, B., Andreasson, J. O. L., Hancock, W. O., \& Block, S. M. (2014). Kinesin processivity is gated by phosphate release. Proceedings of the National Academy of Sciences, 111(39), 14136. https://doi. org/10.1073/pnas.1410943111

Muretta, J. M., Jun, Y., Gross, S. P., Major, J., Thomas, D. D., \& Rosenfeld, S. S. (2015). The structural kinetics of switch-1 and the neck linker explain the functions of kinesin-1 and Eg5. Proceedings of the National Academy of Sciences, 112(48), E6606. https://doi.org/10.1073/pnas.1512305112

Muretta, J. M., Petersen, K. J., \& Thomas, D. D. (2013). Direct real-time detection of the actin-activated power stroke within the myosin catalytic domain. Proceedings of the National Academy of Sciences of the United States of America, 110(18), 7211-7216. https://doi.org/10.1073/pnas. 1222257110

Needham, D. M. (1971). Machina carnis; the biochemistry of muscular contraction in its historical development. University Press.

Palay, S. L. (1956). Synapses in the central nervous system. Journal of Biophysical and Biochemical Cytology, 2(4 Suppl), 193-202.

Rall, J. A. (2014). Mechanism of muscular contraction. Springer. 
Rayment, I., Rypniewski, W. R., Schmidt-Base, K., Smith, R., Tomchick, D. R., Benning, M. M., \& Holden, H. M. (1993). Three-dimensional structure of myosin subfragment-1: A molecular motor. Science, 261(5117), 50. https://doi.org/10.1126/science.8316857

Rice, S., Lin, A. W., Safer, D., Hart, C. L., Naber, N., Carragher, B. O., \& Vale, R. D. (1999). A structural change in the kinesin motor protein that drives motility. Nature, 402(6763), 778-784. https://doi. org/10.1038/45483

Schnapp, B. J., Crise, B., Sheetz, M. P., Reese, T. S., \& Khan, S. (1990). Delayed start-up of kinesindriven microtubule gliding following inhibition by adenosine 5'-[beta, gamma-imido]triphosphate. Proceedings of the National Academy of Sciences, 87(24), 10053. https://doi.org/10.1073/pnas.87. 24.10053

Schnitzer, M. J., \& Block, S. M. (1997). Kinesin hydrolyses one ATP per 8-nm step. Nature, 388, 386. https://doi.org/10.1038/41111

Scholey, J. M., Heuser, J., Yang, J. T., \& Goldstein, L. S. B. (1989). Identification of globular mechanochemical heads of kinesin. Nature, 338(6213), 355-357. https://doi.org/10.1038/338355a0

Sindelar, C. V., \& Liu, D. (2017). Tracking down kinesin's achilles heel with balls of gold. Biophysical Journal, 112(12), 2454-2456. https://doi.org/10.1016/j.bpj.2017.05.008

Smith, C. A., \& Rayment, I. (1995). X-ray structure of the magnesium(II)-pyrophosphate complex of the truncated head of dictyostelium discoideum myosin to $2.7 \AA$ resolution. Biochemistry, 34(28), 8973-8981. https://doi.org/10.1021/bi00028a005

Smith, C. A., \& Rayment, I. (1996). X-ray structure of the magnesium(II).ADP.vanadate complex of the Dictyostelium discoideum myosin motor domain to 1.9 A resolution. Biochemistry, 35(17), 54045417. https://doi.org/10.1021/bi952633+

Svoboda, K., Schmidt, C. F., Schnapp, B. J., \& Block, S. M. (1993). Direct observation of kinesin stepping by optical trapping interferometry. Nature, 365, 722-727.

Szent-Györgyi, A. (1945). Studies on muscle. Arkiv För Kemi, Mineralogi Och Geologi, 19(3), 1-9.

Vale, R. D., \& Milligan, R. A. (2000). The way things move: Looking under the hood of molecular motor proteins. Science, 288(5463), 88-95.

Vale, R. D., Reese, T. S., \& Sheetz, M. P. (1985). Identification of a novel force-generating protein, kinesin, involved in microtubule-based motility. Cell, 42(1), 39-50.

Wang, W., Cao, L., Wang, C., Gigant, B., \& Knossow, M. (2015). Kinesin, 30 years later: Recent insights from structural studies. Protein Science, 24(7), 1047-1056. https://doi.org/10.1002/pro.2697

Winning, J., \& Bechtel, W. (2018). Rethinking causality in neural mechanisms: Constraints and control. Minds and Machines, 28(2), 287-310.

Publisher's Note Springer Nature remains neutral with regard to jurisdictional claims in published maps and institutional affiliations. 